\title{
FLOW INDUCED BY A LINE SINK IN A QUIESCENT FLUID WITH SURFACE-TENSION EFFECTS
}

\author{
LAWRENCE K. FORBES ${ }^{1}$ and GRAEME C. HOCKING ${ }^{2}$
}

(Received 13 March 1991; revised 8 August 1991)

\begin{abstract}
When a line sink is placed beneath the free surface of an otherwise quiescent fluid of infinite depth, two different flow types are now known to be possible. One type of flow involves the fluid being drawn down toward the sink, and in the other type, a stagnation point forms at the surface immediately above the position of the sink.

This paper investigates the second of these two flow types, which involves a free-surface stagnation point. The effects of surface tension are included, and even when small, these are shown to have a very significant effect on the overall solution behaviour. We demonstrate by direct numerical calculation that there are regions of genuine non-uniqueness in the nonlinear solution, when the surfacetension parameter does not vanish. In addition, an asymptotic solution valid for small Froude number is derived.
\end{abstract}

\section{Introduction}

This paper is concerned with determining the steady flow produced by the presence of an isolated line sink, located some distance $H$ beneath the surface of an otherwise stationary fluid. At first sight this problem seems superficially simple, but recent investigations of this deceptive problem reveal a surprising complexity in the solution structure, with many basic questions remaining unanswered.

There are at least two major factors contributing to the unexpected difficulty of this problem. One of these is the fact that, in an ideal fluid, only the

\footnotetext{
${ }^{1}$ Dept. of Math., The University of Queensland, St. Lucia, Qld. 4072, Australia.

${ }^{2}$ Dept. of Math., The University of Western Australia, Nedlands, W. A. 6009, Australia.

(C) Copyright Australian Mathematical Society 1992, Serial-fee code 0334-2700/92
} 
square of the fluid velocity is involved in the formal statement of the problem, so that the solutions are insensitive to the direction of flow along streamlines. Consequently, there is no mechanism for distinguishing between a submerged sink or a source, which introduces the possibility that the problem is in some sense ill-posed. Support for this interpretation might be found from the fact that two quite different types of flow are now known to be possible solutions to the problem. In one of these flow types, the fluid is drawn down to form a vertical cusp above the line singularity, which in this case might be thought of as a line sink. In the other distinct flow type, the fluid gives the appearance of having been pushed out from the line singularity, which consequently might appear to be a source; the fluid surface directly above the singularity has been pushed up to the maximum possible (stagnation) height, at which a surface stagnation point is formed. This appealing interpretation, however, appears to be an over-simplification, since some experiments reported by Imberger [5] showed that a source was capable of producing both of the solution types described above.

The other reason for the inherent difficulty of this problem is the fact that it is highly nonlinear, by virtue both of the defining surface condition (the Bernoulli equation) as well as the fact that the physical location of the free-surface itself is unknown.

The first work on this problem was evidently that of Peregrine [8], who investigated the steady flow produced by a submerged line source in a fluid of infinite depth. He sought a solution using Taylor-series expansions in powers of the square of a Froude number $F$ based on the submergence depth of the line singularity, and so obtained an approximate expression for the surface shape, for the flow type in which a stagnation point appears at the surface. Vanden-Broeck, Schwartz and Tuck [15] have re-examined Peregrine's solution, however, and showed that series expansions in powers of the Froude number are purely divergent. Consequently, Peregrine's solution only retains significance as an asymptotic result. Recently, Hocking [3] and King and Bloor [6] have found a closed-form solution at infinite Froude number for the other type of solution, in which the surface is drawn down into a cusp.

Tuck and Vanden-Broeck [11] used a spectral method in a conformally mapped plane to seek solutions for a submerged line source or sink in a fluid of infinite depth. This approach has subsequently proved very successful in a variety of free-surface flow problems in which surface waves are absent, and has been adopted for example by Hocking and Forbes [4] to compute flow due to a line sink in fluid of infinite depth, and by Hocking [2], Vanden-Broeck and Keller [14] and Mekias and Vanden-Broeck [7] to compute similar flows 
in finite-depth fluid. Tuck and Vanden-Broeck [11] found that they were, in general, unable to compute a solution having a free-surface stagnation point when the Froude number $F$ was free to be specified; some solutions of this type had been obtained for Froude number less than about 2, yet these were ultimately spoiled by the presence of very short-wavelength disturbances at the surface, and were not discussed in great detail by Tuck and VandenBroeck. However, these authors showed that, by allowing the Froude number to be an unknown to be determined as part of the solution, they could obtain a solution of the other type, in which the fluid surface is drawn down toward the sink in a vertical cusped structure. This other solution type occurred at the unique Froude number $F=3.553$.

The method of Tuck and Vanden-Broeck [11] has recently been applied by Hocking and Forbes [4] to the problem of computing the solution type in which a stagnation point is present at the surface of an infinite fluid. They essentially recovered the solutions of Tuck and Vanden-Broeck, but showed that this type of solution is confined to the region $F<1.42$. The shortwavelength disturbances reported by Tuck and Vanden-Broeck were shown to be numerical in origin, and heralded the imminent breakdown of the numerical procedure, apparently corresponding to the actual failure of the nonlinear solution branch itself beyond $F=1.42$. There is no obvious physical reason for the failure of this stagnation-point type of solution beyond $F=1.42$, and the attempted explanation of this behaviour has constituted a partial motivation for undertaking the present study. (In the corresponding three dimensional problem, however, in which a point source is present beneath the surface, Forbes and Hocking [1] found that a similar failure of the branch of solutions possessing a surface stagnation point did in fact have a physical interpretation, and was associated with the formation of a secondary stagnation point on the surface.)

In the present paper, we use a modified form of the method of Tuck and Vanden-Broeck [11] to investigate the flow produced by a line sink in infinitedepth fluid, when the effects of surface tension are taken into account. The problem is stated mathematically in Section 2 of the paper, and the spectral solution technique is outlined. A low-Froude-number asymptotic solution is derived in Section 3. In Section 4, the results of the numerical solution to the fully nonlinear problem are presented and discussed; the inclusion of even a small amount of surface tension has a very significant effect on the solution profiles obtained, which is possibly of some significance for model experimental studies of this type of flow. Additionally, these results suggest a partial explanation of the failure of the solution branch with zero surface tension at the approximate Froude number $F=1.4$. A discussion concludes the paper in Section 5. 
2. Problem statement and spectral solution technique

Consider a quiescent fluid of infinite depth, having density $\rho$ and subject to the downward acceleration $g$ of gravity. The fluid occupies the lower half plane and its surface is a horizontal line. Let us place a Cartesian coordinate system in the fluid, having its $x$-axis along the undisturbed fluid surface (orthogonal to the direction in which gravity acts), and its $y$-axis pointing vertically (in the opposite direction to the gravitational force). The effects of surface tension will be included in the problem, and the surface tension coefficient is denoted by the symbol $T$.

A line sink of strength $m$ per unit width is now introduced into the fluid, at a distance $H$ below the undisturbed fluid surface level; the sink is thus located at the Cartesian point $(0,-H)$. We assume that some steady flow pattern is established, and the shape of the fluid surface alters accordingly. The purpose of this paper is to determine this new surface shape.

Dimensionless variables are now defined, using the sink submergence depth $H$ as the length scale and $m / H$ as the reference speed. The problem then possesses solutions characterised by the two dimensionless parameters

$$
F^{2}=\frac{m^{2}}{g H^{3}} \quad \text { and } \quad \beta=\frac{T}{\rho g H^{2}} .
$$

The first of these constants is the square of the Froude number based on the submergence depth of the line sink beneath the undisturbed surface level, and the second is a dimensionless measure of the importance of surface-tension effects.

We assume that the fluid is ideal, in the sense that it is both incompressible and inviscid. It then follows that the flow occurs without rotation, and the fluid velocity vector with horizontal and vertical components $u$ and $v$, respectively, may be written in terms of a velocity potential $\phi$ and stream function $\psi$, by means of the relations

$$
u=\frac{\partial \phi}{\partial x}=\frac{\partial \psi}{\partial y}, \quad v=\frac{\partial \phi}{\partial y}=-\frac{\partial \psi}{\partial x} .
$$

Equations (2.1) reveal that the complex potential $f=\phi+i \psi$ is an analytic function of the position $z=x+i y$ everywhere in the fluid, except at the line sink where it is singular according to the relation

$$
f \rightarrow-(1 / 2 \pi) \ln (z+i) \text { as } z \rightarrow-i,
$$

since in these dimensionless coordinates, the sink is located at the point $z=-i$.

Suppose that the unknown position of the free surface is denoted as $y(x)$ in the complex $z$-plane. The two conditions to be satisfied at this surface are 
the kinematic constraint

$$
v=u y^{\prime}
$$

which indicates that the surface is a streamline in steady flow, and the Bernoulli equation

$$
\frac{1}{2} F^{2}\left(u^{2}+v^{2}\right)+y-\frac{\beta y^{\prime \prime}}{\left[1+\left(y^{\prime}\right)^{2}\right]^{3 / 2}}=0,
$$

where primes denote differentiation with respect to $x$.

We now follow Tuck and Vanden-Broeck [11] by introducing two conformal transformations to the problem. The first of these is an inversion, in the sense that the complex position $z=x+i y$ is regarded as being dependent upon the potential $f=\phi+i \psi$. This technique was first introduced by Stokes [10], and it has the advantage that the free-surface location is now known in the $f$-plane. In fact, the right half of the flow (which is symmetric about the $y$-axis) maps onto the strip $0 \leq \psi \leq 1 / 2$ in the $f$-plane, with the right half of the free surface occupying the line $\phi \leq 0, \psi=0$. The line sink maps away to $\phi \rightarrow \infty$, which follows from (2.2), and the fluid infinitely far from the sink in the physical $z$-plane is mapped to $\phi \rightarrow-\infty$ in the $f$-plane strip. A further mapping of the $f$-plane into a $t$-plane is also performed, according to the conformal relation

$$
e^{-2 \pi f}=4 t /(t+1)^{2}
$$

The strip $0 \leq \psi \leq 1 / 2$ in the $f$-plane is mapped onto the lower-half of a unit circle in the $t$-plane as a result of (2.5), and the line sink is now located at the origin. The point at infinity in the $z$-plane corresponds to $t=-1$, and the stagnation point which occurs at $z=0$ in the physical plane is mapped to $t=1$. These transformations are similar to those used by Tuck and Vanden-Broeck [11] (apart from a change of sign), and diagrams of the $z$ - $f$ - and $t$-planes are given in their paper, and also appear in Hocking and Forbes [4].

The governing equations are written in the new $t$-plane defined by the mapping (2.5). The conditions (2.1) are equivalent to the statement that $z(t)$ is to be analytic, and the kinematic condition (2.3) is satisfied identically. The sink condition (2.2) becomes simply

$$
z=-i \text { at } t=0,
$$

and the asymptotic form for the outflow of fluid at infinity is

$$
z \rightarrow-\frac{2 i}{t+1} \text { as } t \rightarrow-1 \text {. }
$$

It is convenient to express the independent variable $t$ in polar form $t=r e^{i \theta}$, and then the right half of the free surface of the fluid corresponds to the line 
$r=1,0 \geq \theta \geq-\pi$. After some algebra, the final form of the Bernoulli equation (2.4) is found to be

$$
\frac{F^{2} \sin ^{2} \theta}{8 \pi^{2}(1+\cos \theta)^{2}\left(x_{\theta}^{2}+y_{\theta}^{2}\right)}+y+\frac{\beta\left(x_{\theta} y_{\theta \theta}-y_{\theta} x_{\theta \theta}\right)}{\left(x_{\theta}^{2}+y_{\theta}^{2}\right)^{3 / 2}}=0 \quad \text { on } r=1 \text {. }
$$

We seek a solution to the governing system (2.6)-(2.8) using the spectral representation of Tuck and Vanden-Broeck [11]. The solution $z(t)$ is expressed in the form

$$
z(t)=-\frac{2 i}{t+1} \sum_{j=0}^{\infty} b_{j} t^{j}
$$

with real coefficients $b_{j}$ which are to be determined. This representation (2.9) satisfies condition (2.7), and the sink condition (2.6) at once yields

$$
b_{0}=1 / 2 \text {. }
$$

Tuck and Vanden-Broeck [11] sought a numerical solution to this problem, with $\beta=0$, using a collocation method. If the series (2.9) is approximated with a polynomial in powers of the variable $t$, to order $N$, then there are $N$ coefficients $b_{1}, \ldots, b_{N}$ to be found. Tuck and Vanden-Broeck achieved this by forcing the free-surface condition (2.8) to be satisfied at exactly $N$ points, using Newton's method to accomplish the numerical task. We have likewise tried a collocation approach for the solution of the problem with $\beta=0$, but concerned by the presence of the small free-surface waves that the numerical method may produce, we have also investigated weighted-residual methods as an alternative to collocation. (Tuck has also considered this option, and experimented with least-squares type methods ${ }^{3}$.) It turns out that none of these methods seems to give much improvement over the coefficients $b_{j}$, $j=1, \ldots, N$, obtained by collocation, but possibly the most successful approach we have adopted is a straightforward Galerkin technique, which we now describe.

We express the solution (2.9) in the approximate form

$$
z(t)=x(t)+i y(t)=\sum_{j=0}^{N} b_{j} Z_{j}(t)
$$

with complex basis functions

$$
Z_{j}(t)=X_{j}(t)-i Y_{j}(t)=-2 i t^{j} /(t+1) .
$$

${ }^{3}$ Private Communication. 
On the free surface, $r=1$, the functions $X_{j}$ and $Y_{j}$ may be expressed as

$$
\begin{aligned}
& X_{j}\left(e^{i \theta}\right)=\frac{\sin j \theta+\sin (j-1) \theta}{1+\cos \theta}, \\
& Y_{j}\left(e^{i \theta}\right)=\frac{\cos j \theta+\cos (j-1) \theta}{1+\cos \theta} .
\end{aligned}
$$

The Bernoulli equation (2.8) may now be regarded as a relation of the form

$$
R\left(\theta ; b_{1}, \ldots, b_{N}\right)=0,
$$

where the residual function $R$ is the entire left-hand side of (2.8). The functions $x(\theta), y(\theta)$ and their derivatives appearing in this equation are obtained directly from (2.10a). In the Galerkin approach, the coefficients $b_{1}, \ldots, b_{N}$ are determined (again using Newton's method) so as to satisfy the relations

$$
\begin{aligned}
G_{k}\left(b_{1}, \ldots, b_{N}\right) & =\int_{-\pi}^{0} R\left(\theta ; b_{1}, \ldots, b_{N}\right) Y_{k}\left(e^{i \theta}\right) d \theta \\
& =0, \quad k=1, \ldots, N .
\end{aligned}
$$

The integrals in (2.13) have been evaluated using 256-point Gaussian quadrature, with weights and abscissae taken from the book by Stroud and Secrest [9].

It is desirable to have some measure of the extent of deformation of the free surface. To this end, we introduce the quantity

$$
D=\int_{0}^{\infty}\left[\sqrt{1+\left(\frac{d y}{d x}\right)^{2}}-1\right] d x
$$

which gives the total deflection of the free surface from the horizontal. In the $t$-plane, where the free surface is represented parametrically in terms of the angle $\theta$ on the circumference of the unit circle in the lower-half plane, the expression (2.14) becomes

$$
D=\int_{-\pi}^{0}\left[\sqrt{\left(\frac{d x}{d \theta}\right)^{2}+\left(\frac{d y}{d \theta}\right)^{2}}+\frac{d x}{d \theta}\right] d \theta .
$$

This integral is evaluated easily using Gaussian quadrature, since the numerical grid points in the interval $-\pi \leq \theta \leq 0$ have already been chosen as the appropriate Gaussian abscissae, for the solution of the problem by Galerkin's method.

\section{Asymptotic solution for small Froude number}

In this section, we outline briefly an approximate solution to the problem, valid at low Froude numbers. We recall, however, that the work of Vanden- 
Broeck, Schwartz and Tuck [15] showed that such expansions in powers of the Froude number are divergent; this is presumably the case here also, in spite of the presence of surface-tension effects. Our solution therefore can only be expected to retain significance as an asymptotic approximation.

We express the complex potential $f=\phi+i \psi$ and the surface elevation $y(x)$ in terms of the expansions

$$
\begin{aligned}
& f(z)=f_{0}(z)+O\left(F^{2}\right), \\
& y(x)=F^{2} Y_{1}(x)+O\left(F^{4}\right),
\end{aligned}
$$

and seek to determine the functions $f_{0}(z)$ and $Y_{1}(x)$ by substitution of expressions (3.1) into (2.1)-(2.4).

The zeroth-order complex potential $f_{0}=\phi_{0}+i \psi_{0}$ again satisfies the Cauchy-Riemann equations (2.1) and the sink condition (2.2), but to this order of approximation, the kinematic condition (2.3) becomes simply

$$
\frac{\partial \phi_{0}}{\partial y}=0 \text { on } y=0 \text {. }
$$

The method of images at once gives the solution for $f_{0}(z)$ in the form

$$
f_{0}(z)=-\frac{1}{2 \pi}[\ln (z+i)+\ln (z-i)] \text {. }
$$

The expansions (3.1) and the solution (3.2) are substituted finally into the Bernoulli equation (2.4), and after some algebra, the function $Y_{1}(x)$ is found to satisfy the relation

$$
\beta Y_{1}^{\prime \prime}(x)-Y_{1}(x)=\frac{x^{2}}{2 \pi^{2}\left(x^{2}+1\right)^{2}} .
$$

Equation (3.3) is a straightforward second-order differential equation, and can be solved using variation of parameters. After some calculation, the solution may eventually be expressed in the form

$$
Y_{1}(x)=-\frac{1}{4 \sqrt{\beta} \pi^{2}}\left[\int_{0}^{\infty} \frac{e^{-\xi / \sqrt{\beta}}(\xi+x)^{2}}{\left[(\xi+x)^{2}+1\right]^{2}} d \xi+\int_{0}^{\infty} \frac{e^{-\xi / \sqrt{\beta}}(\xi-x)^{2}}{\left[(\xi-x)^{2}+1\right]^{2}} d \xi\right] .
$$

We have evaluated these integrals numerically using 68-th order GaussLaguerre quadrature, with coefficients again taken from the book by Stroud and Secrest [9]. It is easy to verify that the solution (3.4) is symmetric about the $y$-axis, and that its slope is zero at $x=0$, but notice now that the stagnation point (at $x=0$ ) does not occur at the stagnation height $y=0$ but at some lower surface elevation. 
When surface tension is absent, $\beta=0$, the surface elevation can be obtained as the limit of (3.4). Combining the result with (3.1) then yields

$$
y(x)=-\frac{F^{2} x^{2}}{2 \pi^{2}\left(x^{2}+1\right)^{2}}+O\left(F^{4}\right) .
$$

In this simple case, the deflection can be worked out in closed form from the definition (2.14). We obtain

$$
D=\frac{F^{4}}{2 \pi^{4}} \int_{0}^{\infty} \frac{x^{2}\left(1-x^{2}\right)^{2}}{\left(x^{2}+1\right)^{6}} d x+O\left(F^{6}\right)
$$

which can be evaluated using the calculus of residues to yield the simple result

$$
D=\frac{F^{4}}{256 \pi^{3}}+O\left(F^{6}\right)
$$

We have not obtained a closed form result for this quantity when $\beta \neq 0$, however.

\section{Presentation of results}

We begin this section by investigating the dependence of the nonlinear solutions upon the surface-tension parameter $\beta$. Some results of this type are shown in Figure 1, in which the deflection $D$, computed from (2.15), is

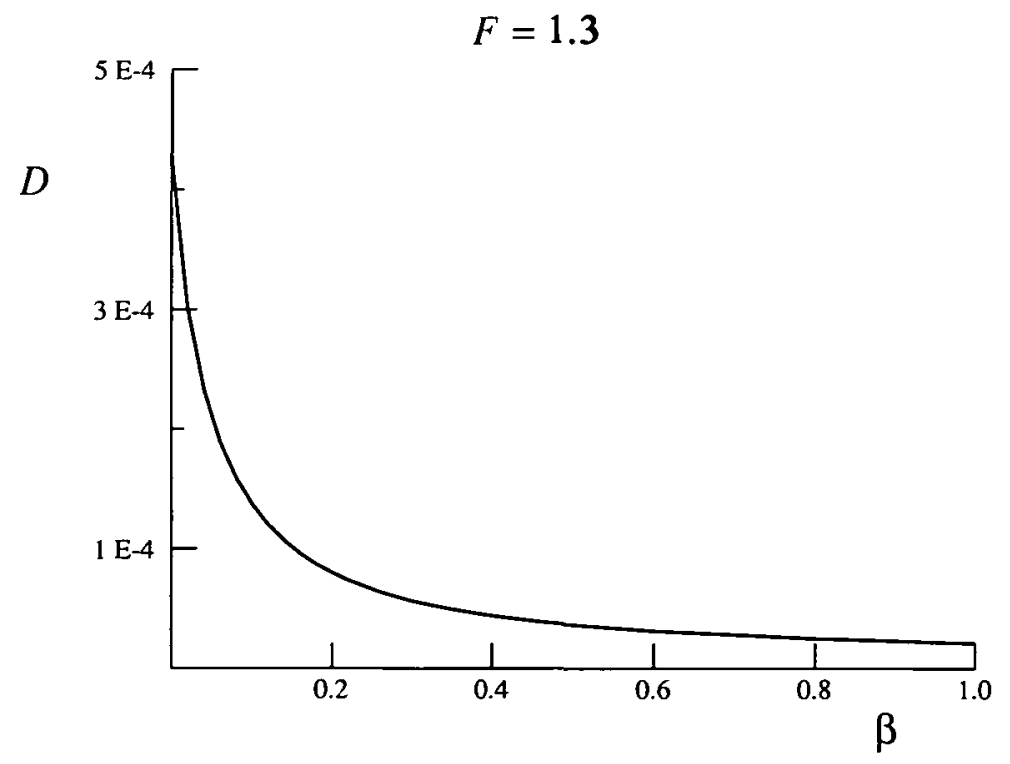

FIGURE 1. The dependence of surface deflection $D$ upon the surface-tension parameter $\beta$, at Froude number $F=1.3$. 


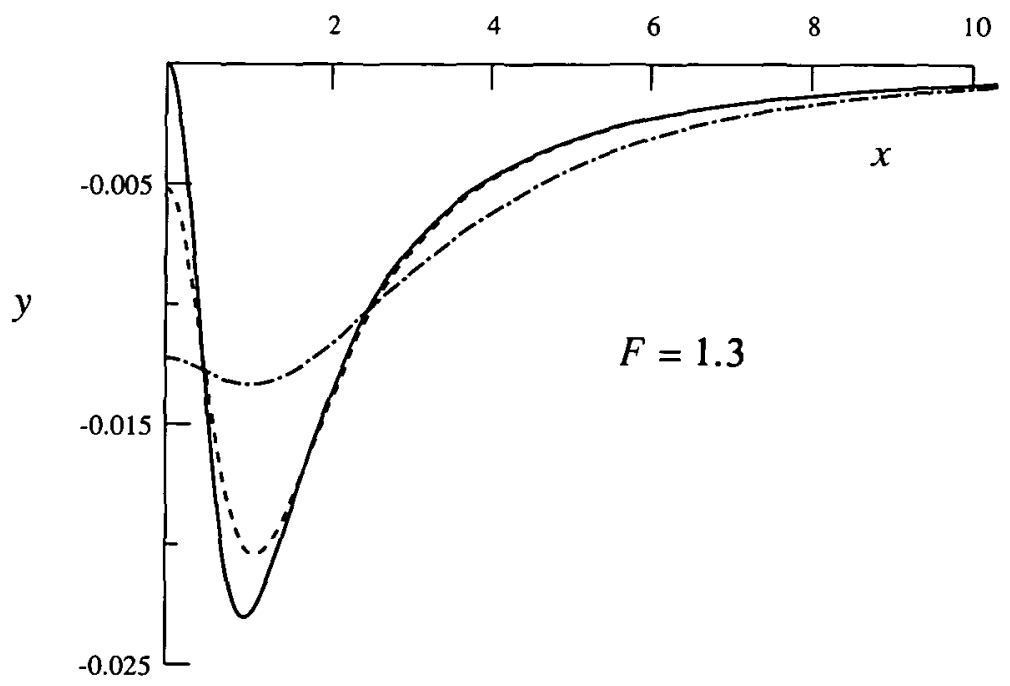

FIGURE 2. Three free-surface profiles obtained at Froude number $F=1.3$, at values of the surface-tension parameter $\beta=0(-), \beta=0.06(---)$, and $\beta=2(-\cdot-\cdot)$.

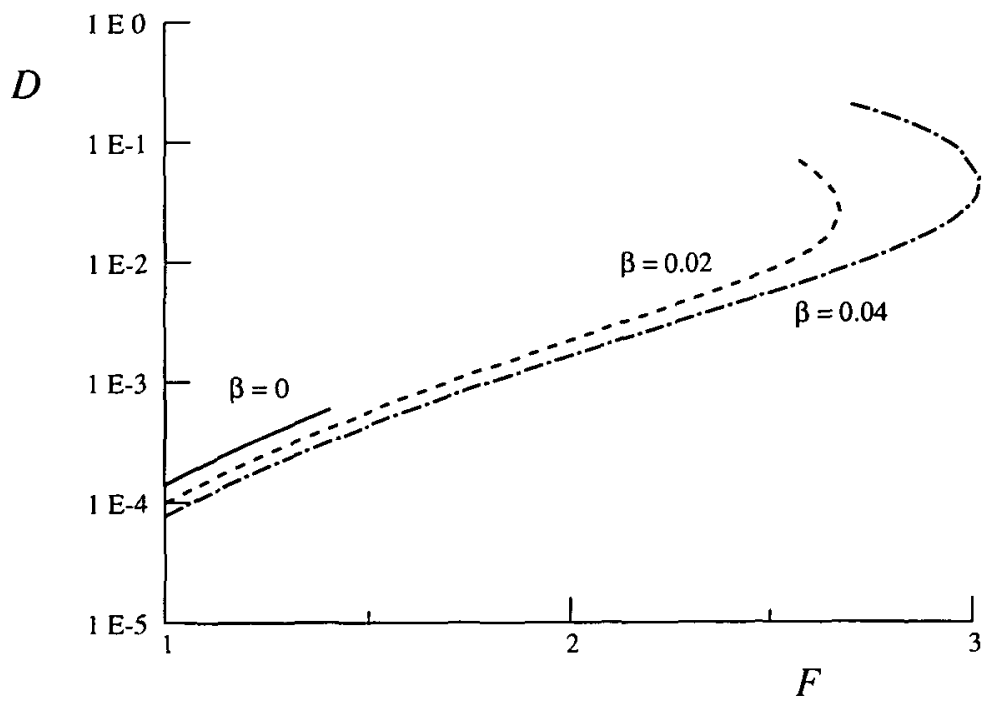

FIGURE 3. The dependence of surface deflection $D$ upon the Froude number $F$, at the three different values of the surface-tension parameter $\beta=0, \beta=0.02$ and $\beta=0.04$. This figure summarises the results of over 100 separate converged solutions. Notice that the scale on the ordinate is logarithmic. 
displayed as a function of $\beta$, for a Froude number $F=1.3$. Recall that, with zero surface tension, the maximum possible Froude number at which these stagnation-point type solutions exist has been computed by Hocking and Forbes [4] to be about $F=1.4$, and so the solution in Figure 1 with $\beta=0$ is reasonably close to this maximum. As the surface tension is increased, the deflection $D$ diminishes at first rapidly, and the surface must therefore deviate less from the undisturbed level $y=0$.

This is confirmed in Figure 2, where three different surface profiles are shown for $F=1.3$. The solid line is the zero surface tension result of Hocking and Forbes [4] $(\beta=0)$, the dashed line corresponds to $\beta=0.06$ and the broken line represents the surface shape for $\beta=2$. The maximum deviation of the surface below the horizontal (which occurs at about $x=1$ ) is diminished as $\beta$ is increased, although the stagnation point at $x=0$ continues to move away from the origin.

The major results of this paper are those given in Figure 3. Here, the dependence of the deflection $D$ upon the Froude number $F$ is shown for three different values of the surface-tension parameter $\beta$. The solid line represents the results obtained for $\beta=0$; as observed by Hocking and Forbes [4], it terminates at about $F=1.4$ for a reason which is not well understood. The dashed line corresponds to results obtained with $\beta=0.02$ and the broken line is the deflection curve for $\beta=0.04$.

In the absence of surface tension $(\beta=0)$, the deflection increases with increasing Froude number, reaching a maximum at about $F=1.4$. Beyond this value, the numerical method ultimately fails to yield a solution, in the sense described by Hocking and Forbes [4]. When the number of coefficients $N$ in the spectral representation $(2.10 \mathrm{a})$ is small, the Newton's method technique converges to a free-surface shape which contains nonphysical wavelets, for $F>1.4$. As $N$ is increased, however, the surface profile does not become independent of $N$, and eventually the Newton algorithm fails to yield a solution at all for sufficiently large $N$. When $F<1.4$, these difficulties do not occur, and highly converged surface profiles have been obtained.

When the surface tension is not zero, the deflection increases with increasing Froude number, until some maximum Froude number is attained. At this point, the deflection curves actually undergo a fold, so that there is an interval of Froude numbers within which two different solutions can exist simultaneously. This behavior is evident in Figure 3, for the two curves obtained with $\beta=0.02$ and $\beta=0.04$. As the surface-tension parameter $\beta$ is decreased, the maximum Froude number (at which the fold occurs) likewise decreases. It is presumably the case that the solution branch before the fold is reached is stable, and that beyond the fold is possibly unstable, although this conjecture could only be confirmed by an investigation of the full time-dependent problem. 


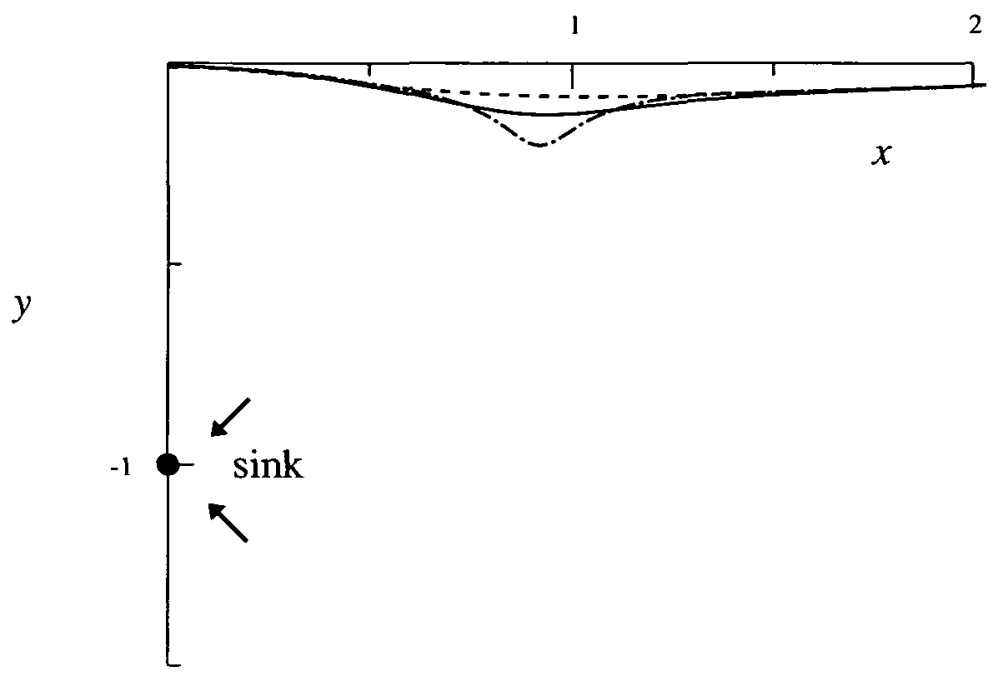

(a)

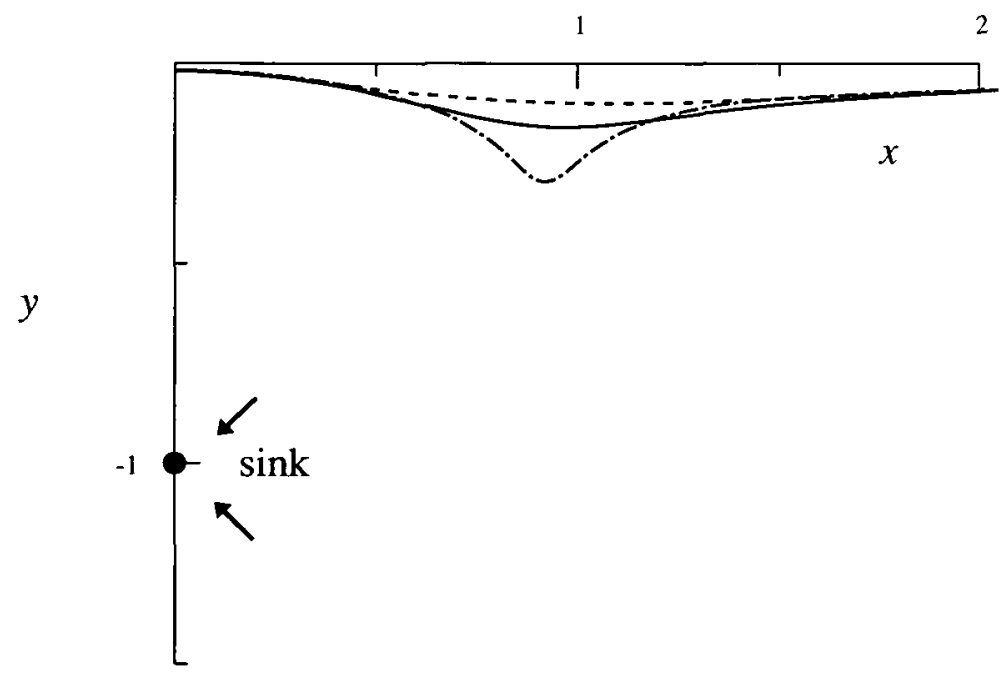

(b)

FIGURE 4. Free-surface profile predicted by the asymptotic solution of Section $3(---)$, and non-unique profiles computed numerically at a point below the fold singularity $(-)$ and above the fold (-.-.-). Results are shown for (a) $F=2.6, \beta=0.02$, and for (b) $F=2.9$, $\beta=0.04$. The scale on the vertical and horizontal axes is the same, and the location of the line sink is shown. 
The solution branches in Figure 3 were continued around the fold singularities using a modified numerical method, in which the first Fourier coefficient $b_{1}$ in equation (2.10a) was given in advance, and the Froude number $F$ allowed to be determined as part of the solution. Slightly beyond the fold, however, the numerical results appear to break down in the sense of Hocking and Forbes [4], for a reason that is again not clear.

Non-unique surface profiles are displayed in Figures 4; these are a consequence of the fold singularities encountered in Figure 3. In Figure 4(a), results are displayed for the case $F=2.6, \beta=0.02$. The solid line is the surface profile corresponding to the portion of the curve in Figure 3 before the fold had been reached, and the broken line is the (presumably unstable) solution in the portion of the branch beyond the fold singularity. The dashed line is the surface profile predicted by the asymptotic solution (3.1) and (3.4), and it gives surprisingly good agreement with the nonlinear solutions for $x>2$, even in these extreme cases. The situation in Figure 4(b) is similar, and shows surface profiles for the case $F=2.9, \beta=0.04$. The solid line is again the solution below the fold singularity and the broken line is the solution beyond it. The dashed line represents the predictions of the asymptotic theory of Section 3, and again is in good agreement with the nonlinear results for $x>2$.

\section{Summary and discussion}

This paper continues the work of Tuck and Vanden-Broeck [11] and Hocking and Forbes [4] on stagnation-point type flow caused by a line sink or source beneath the free surface of otherwise stationary fluid. The solution technique has again made extensive use of conformal mappings, and involves a Galerkin method. The results of Hocking and Forbes have been recovered for zero surface tension, and show that the method fails for Froude numbers $F$ greater than about 1.4 . For nonzero surface tension $\beta$, there is a maximum possible Froude number at which solutions of this type can be achieved, and this maximum is associated with the formation of a fold in the bifurcation diagram. There is thus an interval of Froude numbers slightly below this maximum, within which two different solutions are possible.

Even a small amount of surface tension can evidently have significant effects on the overall solution behaviour. In particular, the maximum permissible Froude number is very sensitive to this parameter, which could have a major influence on a model experiment where the effects of surface tension were ignored. Thus the maximum Froude number $F=1.4$ for zero surface tension, reported by Hocking and Forbes [4], is already increased to about $F=2.67$ for $\beta=0.02$. 
Our original motivation for investigating this problem, in which surfacetension effects are included, was strongly influenced by the desire to explain the failure of the solution branch at $F=1.4$ with zero surface tension, reported by Hocking and Forbes [4]. Perhaps some partial success in this aim has been achieved. When surface-tension effects are included, the deflection curves in Figure 3 undergo a fold at the maximum Froude number; as the surface-tension parameter $\beta$ is reduced, both the strength of the fold and the value of the maximum Froude number decrease. In the limit $\beta \rightarrow 0$, it seems that the maximum Froude number decreases to 1.4, at which point some sort of degenerate fold singularity is evidently present. It appears that steady solutions are no longer possible past this point.

We have not commented in this paper on the other type of flow possible in this situation, in which, in the absence of surface tension, the surface is drawn down into a cusp, as described by Tuck and Vanden-Broeck [11]. It is to be expected that this cusped solution at zero surface tension may be continued analytically to parameter values involving nonzero surface tension, although the cusp will presumably be replaced by a finite-angle wedge at the surface, immediately above the line sink. In addition, when surface tension effects are present, there is available a second, meniscus-type solution at zero Froude number, in addition to that discussed in Section 3. This solution also involves a free-surface wedge of finite angle, and presumably may also be continued numerically to nonzero values of the Froude number. A consideration of these additional solution types is beyond the scope of the present paper, and work is currently in progress to compute such flows, using methods similar to those of Vanden-Broeck [12], [13] in which the unknown surface angle at the singular point is computed as part of the solution.

\section{References}

[1] L. K. Forbes and G. C. Hocking, "Flow caused by a point sink in a fluid having a free surface", J. Austral. Math. Soc. Ser. B 32 (1990) 231-249.

[2] G. C. Hocking, "Cusp-like free-surface flows due to a submerged source or sink in the presence of a flat or sloping bottom", J. Austral. Math. Soc. Ser. B 26 (1985) 470-486.

[3] G. C. Hocking, "Infinite Froude number solutions to the problem of a submerged source or sink", J. Austral. Math. Soc. Ser. B 29 (1988) 401-409.

[4] G. C. Hocking and L. K. Forbes, "A note on the flow induced by a line sink beneath a free surface", J. Austral. Math. Soc. Ser. B 32 (1991) 251-260.

[5] J. Imberger, "Selective withdrawal: a review", 2nd International Symposium on Stratified Flows, Trondheim, Norway, 1980.

[6] A. C. King and M. I. G. Bloor, "A note on the free surface induced by a submerged source at infinite Froude number", J. Austral. Math. Soc. Ser. B 30 (1988) 147-156.

[7] H. Mekias and J.-M. Vanden-Broeck, "Supercritical free-surface flow with a stagnation point due to a submerged source”, Phys. Fluids, Ser. A 1 (1989) 1694-1697. 
[8] D. H. Peregrine, "A line source beneath a free surface", Univ. Wisconsin Math. Res. Center Tech. Summ. Report 1248 (1972).

[9] A. H. Stroud and D. Secrest, Gaussian quadrature formulas, (Prentice-Hall, Inc., Englewood Cliffs, N.J., 1966).

[10] G. G. Stokes, Mathematical and Physical Papers, Vol. 1, (Cambridge University Press, 1880).

[11] E. O. Tuck and J.-M. Vanden-Broeck, “A cusp-like free-surface flow due to a submerged source or sink", J. Austral. Math. Soc. Ser. B 25 (1984) 443-450.

[12] J.-M. Vanden-Broeck, "The effects of surface tension on the shape of the Kirchhoff jet", Phys. Fluids 27 (1984) 1933-1936.

[13] J.-M. Vanden-Broeck, "Rising bubbles in a two-dimensional tube with surface tension", Phys. Fluids 27 (1984) 2604-2607.

[14] J.-M. Vanden-Broeck and J. B. Keller, "Free surface flow due to a sink", J. Fluid Mech. 175 (1987) 109-117.

[15] J.-M. Vanden-Broeck, L. W. Schwartz and E. O. Tuck, "Divergent low-Froude-number series expansion of nonlinear free-surface flow problems", Proc. Roy. Soc. London, Ser. A 361 (1978) 207-224. 